\title{
El papel del tutor en la elaboración de trabajos de tesis
}

\section{The role of the tutor in the elaboration of thesis work}

Augusto César García Duarte

Recibido: 30 de enero de 2019, Aceptado: 20 de mayo de 2019

\section{RESUMEN}

Una de las formas de graduación, de la Universidad Nacional Autónoma de Nicaragua y particularmente quiero referirme a su Facultad Regional Multidisciplinaria-Estelí, es la elaboración de un tesis, sea este propuesto por el estudiante o mediante la asignatura de seminario de graduación. Esto conlleva la elaboración de un documento científico que aborda una problemática y generalmente ofrece una solución al mismo. Para esto se designa una figura denominada tutor.

Palabras claves: formas de graduación; tutor.

\section{ABSTRACT}

One of the graduation options, of the National Autonomous University of Nicaragua and particularly I want to refer to its Multidisciplinary Regional Faculty of Estelí, is the elaboration of a thesis, either this proposed by the student or through the subject of Graduation Seminar. This involves writing a scientific document that addresses a problem and usually offers a solution to it. For this a figure called tutor is designated.

Keywords: graduation options; tutor.

1 Docente de UNAN-Managua/FAREM-Estelí. Correo electrónico: augustogarciaduarte@yahoo.com.

(c) 2019 Revista Multi-Ensayos. 


\section{INTRODUCCIÓN}

Según la Real Academia Española (Española, 2017), define el concepto de tutor de la siguiente forma:

Del lat. tutor, -ōris.

1. m. y f. Persona que ejerce la tutela.

2. m. y f. Persona encargada de orientar a los alumnos de un curso o asignatura.

3. m. y f. Defensor, protector o director en cualquier línea.

4. m. y f. Persona que ejerce las funciones señaladas por la legislación antigua al curador.

5. m. y f. Profesor privado que se encargaba de la educación general de los hijos de una familia.

6. m. Caña o estaca que se clava al pie de una planta para mantenerla derecha en su crecimiento.

La conceptualización del ser una persona encargada de "orientar", es decir está limitado a una función orientadora o de guía del proceso de investigación y elaboración de documentos de los trabajos de los estudiantes. Pero se podrá detallar de manera puntual algunas funciones del tutor, que tipo de guía es efectiva para finalizar de manera exitosa la investigación. (Blanco Fernández, 2009)

\section{DESARROLLO}

Para Difabio de Anglat (2011), las funciones que debería desarrollar un tutor de tesis doctorales, las cuales según mi criterio con aplicables a todos los trabajos investigativos sin importar el grado al que se haga referencia, son:

- Evaluación temprana y realista de las necesidades del estudiante: Determinar de manera puntual los conocimientos previos del estudiante en dos aspectos, el primero el proceso de investigación y el segundo la temática que se desea investigar. Esto nos dará insumos para fortalecer esas carencias y orientar las lecturas indicadas que permitan complementar y asegurar el aprendizaje.

- Foco pedagógico: No debemos olvidar que este proceso de tutoría también sirve de aprendizaje, tanto para el tutor como para el estudiante investigador, lo que permitirá construir nuevo conocimiento, siendo una oportunidad de autoformación para ambos actores de los procesos investigativo.

- Clarificación de expectativas y límites: Es vital en un momento inicial, una conversación que permita sentar las bases del proceso de tutoría, dejando claro las funciones tanto del tutor como del estudiante, los días de reunión, que se espera realizar en cada una de dichas reuniones. Cabe mencionar, que siendo el proceso de investigación un proceso dinámico y cambiante durante el proceso es necesario evaluar de manera permanente los acuerdos tomados por ambas partes y realizar modificaciones según lo estimen conveniente.

- Desafío académico y valoración: Las reuniones consensuadas deben servir para evaluar de manera crítica el proceso de investigación que se está realizando, siendo papel del tutor ser claro y criticarlo de manera constructiva. Además, debe realizarse de manera oportuna, no dejando pasar el tiempo, puesto que esto acarrea problemas que crecerán al pasar con el tiempo.

- Gestión del conocimiento: Los tutores deberán dominar las temáticas a investigarse, pero siendo que no lo conocemos todos, es mucho más importante que el tutor sea capaz de orientar al estudiante en las temáticas vitales para su investigación, acá vemos el proceso de aprendizaje de ambos actores del proceso. Otro elemento a considerar es promover la participación del estudiante en eventos de investigadores como por ejemplo la Jornada de Investigación Científica que se realiza en la UNANManagua.

- Frecuencia y calidad de las reuniones: Las reuniones planificadas, no deben ser un mero cumplimiento de horas asignadas, sino más bien una oportunidad para mejorar la calidad del trabajo de investigación, esto mediante una orientación oportuna y adecuada. Es decir que las reuniones deben tener un objetivo o propósito, planificadas de manera anticipada por el tutor tanto en contenido como en 
tiempo, esto permitirá realizar las adecuaciones en tiempo y forma.

- Revisión del trabajo escrito: El tutor no debe omitir la lectura del documento elaborado por los estudiantes, tampoco es una tarea que debe dejarse para el final del proceso. Asimismo, debemos realizar las lecturas de manera sistemática, lo que permite revisar, corregir el documento paso a paso, detectando y corrigiendo de manera oportuna cualquier error.

- Feedback inmediato y constructivo: En cada una de las reuniones, debemos realizar una evaluación del proceso, tanto de proceso como documental, proveyendo una retroalimentación inmediata y crítica pero de manera constructiva, pero no únicamente el criticar el proceso de parte de los estudiantes sino que debe ser autocritico en el proceso de tutorización que se está realizando. También debemos permitir al estudiante que evalúe el proceso y proponga cambios.

- Advertencia de las señales de peligro: El tutor debe esta alerta a situaciones que pongan en riesgo el proceso investigativo, retardo en la captación de información, falta de documentar lo realizado, todo esto amerita un ajuste de parte del tutor para evitar que la investigación decaiga o pierda relevancia.

Siendo críticos estas funciones se cumplen en su gran mayoría en la tutorización de trabajos de tesis, quizás lo único que faltaría por realizar es dar a conocerlas a los estudiantes. Es decir en una primera conversación poner las cartas sobre la mesa, indicando las tareas de cada una de las partes involucradas.

\section{CONCLUSIÓN}

En los trabajos de investigación, es común encontrarse con quejas de parte de los estudiantes de lo que ellos definen como una falta de tutoría adecuada. En ocasiones nuestro papel de tutor no es percibido como tal, es decir, los estudiantes pretenden que el tutor realice ciertas tareas que son propias del investigador, me refiero a redactar objetivos, elaborar tablas, cuadros y cualquier otro tipo de redacción. Por otra parte el tutor se queja de los investigadores de no avanzar o no realizar lo que el oriento en una reunión previa.

Por lo antes mencionado, es necesario dejar claro desde un primer momento las funciones de cada uno de los participantes, realizar un cronograma de trabajo, definiendo los tiempos de realización de cada uno de las etapas de la investigación.

Las tareas del tutor consisten en: detectar las dificultades de los estudiantes tanto a nivel investigativo como de la redacción del documento, además debe encauzar el curso del proceso cuando este se desvié o se estanque. Asimismo, debe criticar el proceso de manera enérgica pero constructiva, facilitando el proceso de retroalimentación de manera oportuno y permitir las modificaciones a las tareas establecidas siempre y cuando se considere pertinente y necesario.

Por ultimo pero no menos importante, el tutor no debe olvidar que este proceso de investigación también es un proceso de aprendizaje para los participantes. Así mismo, tener en cuenta que los autores de la investigación son los estudiantes, su función es meramente la que "orientar" y llevar a puerto seguro dicho proceso.

\section{REFERENCIAS}

Blanco Fernández, A. (2009). Desarrollo y evaluación de competencias en educación superior. España: Narcea S.A ediciones.

Difabio de Anglat, H. (2011). Las funciones del tutor de la tesis doctoral en educaciòn. Revista mexicana de investigación educativa, 935-959.

Real Academia Española. (01 de 10 de 2017). Diccionario de la Lengua Española. Recuperado el 01 de 10 de 2017, de Diccionario de la Lengua Española: http://dle.rae.es/?id=aze4nV6 